\title{
High throughput array technologies: Expanding applications from clinics to applied research
}

\author{
Ajay Vikram Singh ${ }^{1,2^{*}}$, Donato Gemmati ${ }^{3}$, Tanushree Vats ${ }^{4}$, Archana Singh ${ }^{5}$ and Paolo Zamboni ${ }^{6}$ \\ ${ }^{1}$ Department of Chemical and Product Safety, German Federal Institute for Risk Assessment (BfR), Max-Dohrn-Strasse 8-10, 10589, Berlin, Germany \\ ${ }^{2}$ Physical Intelligence Department, Max Planck Institute for Intelligent Systems, 70569 Stuttgart, Germany \\ ${ }^{3}$ Hemostasis \& Thrombosis Center - Azienda Ospedaliera-Universitaria di Ferrara, Ferrara, Italy \\ ${ }^{4}$ Department of Microbiology, Sahara Hospital, Gomti Nagar, Lucknow 226010 (UP) India \\ ${ }^{5}$ KNIPSS Managmeent Insitute, Faridipur Campus, NH 96, Faizabad-Allahabad Rd., Sultanpur 228119 (UP) \\ ${ }^{6}$ Translational Surgery Unit, Azienda Ospedaliera Universitaria di Ferrara, via Aldo Moro 844124 Ferrara Italy
}

\section{Commentary}

The electronic micro-nanoarray technology is providing accountable contribution in field of clinical diagnosis and biological research [1]. Currently, based on fine tools and aid supported by nanotechnology and nanochip patterning advancement [2,3], micro-nanoarray is revolutionizing molecular biology and genetics research. Utilizing best optical detection methods and DNA chemistry detecting changes in genomics/transcriptomics and more challenging, proteomics via hybridization and fine optical detection at nanochip, micro-nanoarray technology going beyond the expectation in the field of current research. Henceforth this technology is delivering the best output to look into individual gene and its subsequent mutation that affects population towards debilitating neurological disorders and imposes genetic susceptibility in disease prognosis [4].

Recently, based on the advances in machine learning, there has been tremendous spurge in the field of micro-nanorobotics to complex data analysis revealing new insight into fundamental biology [5-8]. In this regard, potential application set forth by electronic micronanoarray ranges in detecting point mutations to single nucleotide polymorphisms (SNPs) in human genome relevant to disease diagnosis in oncogene SNParray, indications to predisposition of cancer to population, responses to chemotherapeutics and associated toxicity helping out best way cancer treatment and diagnosis.

Other associated partners benefiting maximum with electronic micro-nanoarray technology are neurodegenerative disorders including Alzheimer diseases (AD), Parkinson (PD) and multiple Sclerosis (MS) [9]. In past few years genetic susceptibility in Neurodegenerative disorders, Venous Leg ulcer (VLU) and Cardio vascular diseases associated with Iron over load gene polymorphism (MMPs, HFE, FPN, HEPC, Tf etc.) had recalled much interest of clinicians and researches to evaluate genetic susceptibility associated with polymorphic gene profile. Electronic micro-nanoarray contributed a lot in this view as quick and accurate assessment of genetic eclipse if any on pathophysiology of diseases.

Micro-nanoarray by definition comprises, exact point placement of numerous DNA samples on the smallest area. For this, oligonucleotides, which represent the genes to be examined, are placed with high precision onto a glass slide (spotting). In the hybridization process that follows, these oligonucleotides identify and link the corresponding structure in the sample. The components of a complete system can be divided into three parts as given blow in Figure 1 sample preparation, array generation and sample analysis.

The focus area of micro-nanoarray investigation in our lab are the association of SNPs those increase susceptibility and progression of MS and VLU related with iron homeostasis gene. Among various factors related with MS and VLU, we projected our studies related with possible associations between HFE genes (H63D, C282Y) and -8CG SNP in the promoter of the ferroportin (FPN1) gene in MS. For VLU, we considered FXIII V34L, MMP-12, FPN1 -8C/G and HFE C282Y as candidate gene for polymorphism $[10,11]$. The above recommendation of candidate genes were made considering their vital role in iron metabolism and homeostasis and to investigate any probable role in diagnosis, prognosis and prevention of MS and ulcer [12]. Specially, there is scarcity of available research regarding the role of FPN1 gene polymorphism in MS or associated neurodegenerative disorders though it plays a critical role in iron export outside the cells [13-15].

\section{Acknowledgement}

AVS thanks Max Planck Institute for Intelligent Systems for the grass root project grants in 2017 (M10335) and 2018 (M10338).

\section{Conflict of Interest}

Authors state no conflict of interests.

${ }^{*}$ Correspondence to: Singh AV, Department of Chemical and Product Safety, German Federal Institute for Risk Assessment (BfR), Max-Dohrn-Strasse 8-10, 10589, Berlin, Germany and Physical Intelligence Department, Max Planck Institute for Intelligent Systems, 70569 Stuttgart, Germany; Email: avsingh@is.mpg.de

Special Issue: Nanotechnology: Challenges and Perspectives in Medicine

Dr. Federica Valentini

Department of Sciences and Chemical Technologies

Tor Vergata University

Italy

Maurizio Talamo

Professor

Department of Enterprise Engineering

Italy

Received: May 01, 2019; Accepted: May 07, 2019; Published: May 09, 2019 

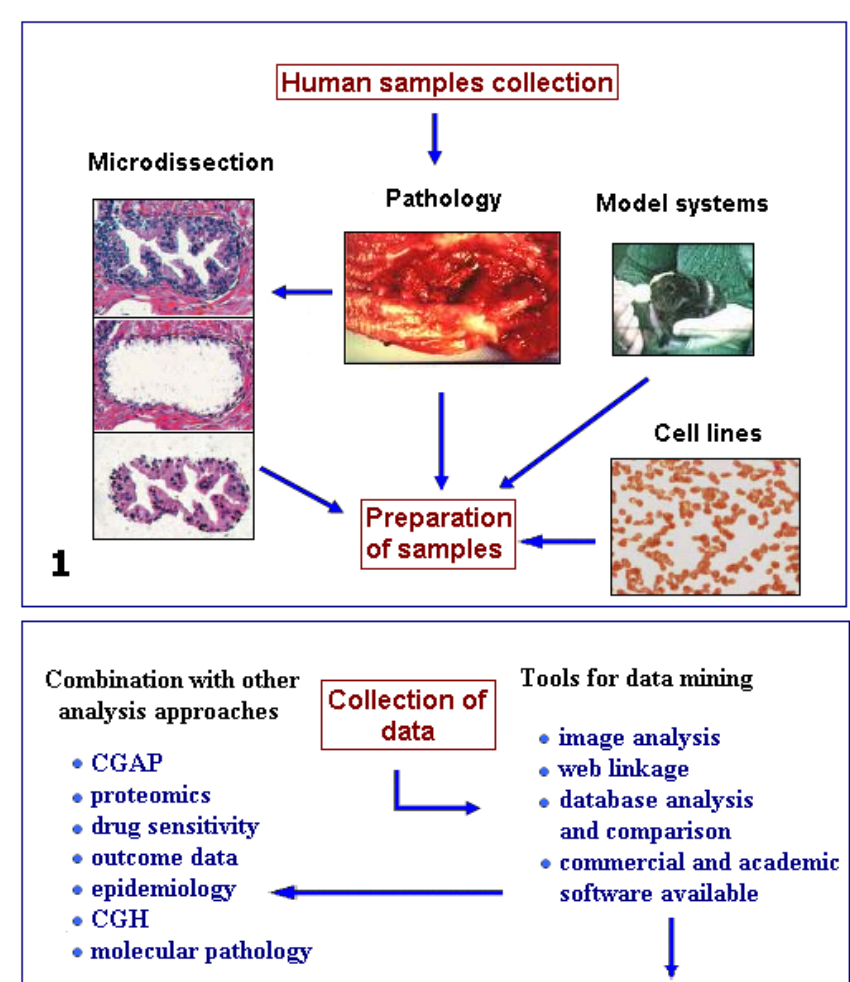

3

Problems in analysis pattern recognition

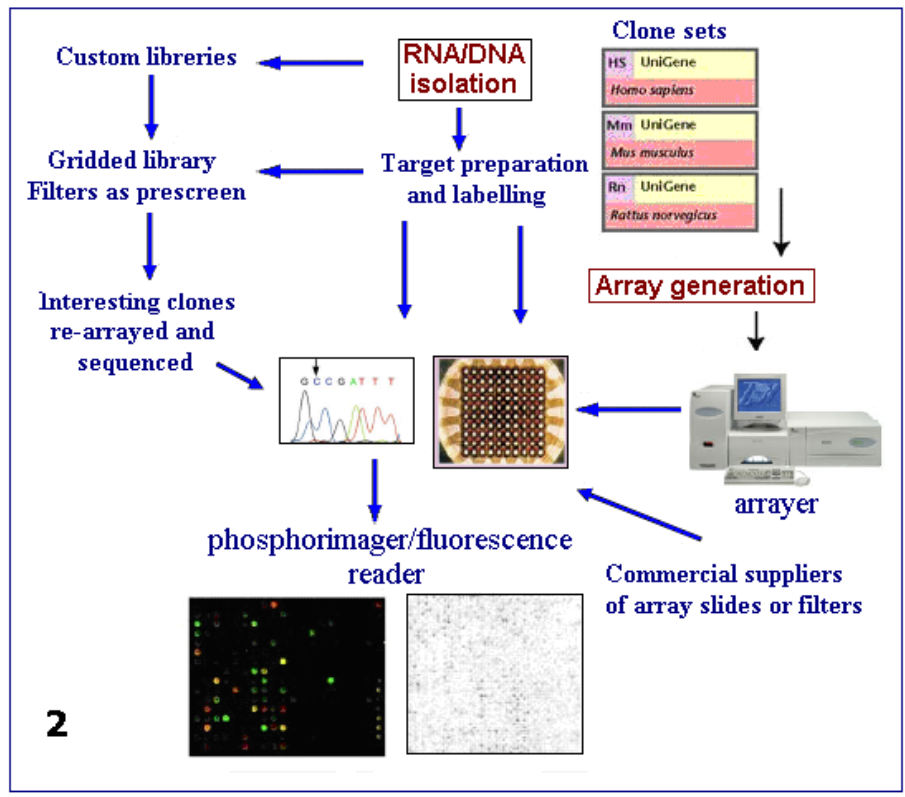

Figure 1. Sample preparation, array generation and sample analysis.

\section{References}

1. Kononen J, Bubendorf L, Kallionimeni A, Bärlund M, Schraml P. et al. (1998) Tissue microarrays for high-throughput molecular profiling of tumor specimens. Nat Med 4: 844.

2. Singh AV, Gharat T, Batuwangala M, Park BW, Endlein T, et al. (2018) Three-dimensional patterning in biomedicine: Importance and applications in neuropharmacology. J Biomed Mater Res B Appl Biomater. 106: 1369-1382.

3. Singh AV, Ansari MHD, Wang S, Laux P, Luch A, et al. (2019) The adoption of three-dimensional additive manufacturing from biomedical material design to $3 \mathrm{~d}$ organ printing. Applied Sciences 9: 811.

4. Paraboschi EM, Cardamone G, Rimoldi V, Gemmati D, Spreafico M, et al. (2015) Meta-analysis of multiple sclerosis microarray data reveals dysregulation in rna splicing regulatory genes. Int J Molecular Sci 16: 23463-23481.

5. Singh SP, Rathee N, Gupta H, Zamboni P, Singh AV (2017) Contactless and hassle free real time heart rate measurement with facial video. J Card Crit Care 1: 024-029.

6. Singh AV, Jahnke T, Wang S, Xiao Y, Alapan Y, et al. (2018) Anisotropic gold nanostructures: Optimization via in silico modeling for hyperthermia. ACS Applied Nano Materials 1: 6205-6216.

7. Singh AV, Sitti M (2016) Bacteria-driven particles: Patterned and specific attachment of bacteria on biohybrid bacteria-driven microswimmers (adv. Healthcare mater. 18/2016). Adv Healthc Mater 5: 2306-2306.
8. Raymond MJ, Ray P, Kaur G, Fredericks M, Singh AV, et al. (2017) Multiaxial polarity determines individual cellular and nuclear chirality. Cell Mol Bioeng 10: 63-74.

9. Singh AV (2010) Multiple sclerosis takes venous route: Ccsvi and liberation therapy Indian J Med Sci 64: 337-337.

10. Singh AV (2007) Micro-array project for diagnosis of vascular lesions under the topic nanotechnology and nanoscience. University of Ferrara.

11. Sheykhansari S, Kozielski K, Bill J, Sitti M, Gemmati D, et al. (2018) Redox metals homeostasis in multiple sclerosis and amyotrophic lateral sclerosis: A review. Cell Death Dis 9: 348.

12. Singh AV, Gemmate D, Kanase A, Pandey I, Misra V, et al. (2018) Nanobiomaterials for vascular biology and wound management: A review. Veins and Lymphatics 7.

13. Lanzara S, Catozzi L, Palma MD, Federici F, Palazzo A, et al. (2006) Mechanism of disease: Inflammatory chain in chronic venous disorders and genetic screening for prevention of venous leg ulcers. Acta Phlebologica 7:39-44.

14. Dwivedi C, Pandey I, Pandey H, Patil S, Mishra SB, et al. (2018) In vivo diabetic wound healing with nanofibrous scaffolds modified with gentamicin and recombinant human epidermal growth factor. $J$ Biomed Mater Res A 106: 641-651.

15. Dwivedi C, Pandey H, Pandey AC, Patil S, Ramteke PW, et al. (2019) In vivo biocompatibility of electrospun biodegradable dual carrier (antibiotic + growth factor) in a mouse model-implications for rapid wound healing. Pharmaceutics 11: 180.

Copyright: (C2019 Singh VA. This is an open-access article distributed under the terms of the Creative Commons Attribution License, which permits unrestricted use, distribution, and reproduction in any medium, provided the original author and source are credited. 